\section{HOSPITAL E INSTITUTO DE SANIDAD DEL CENTRO MEDICO DE DETROIT MICHIGAN/EE. UU.}

\author{
William Kessler, Arquitectos y Asociados \\ Compañía Zeidler \\ Giffels Asociados \\ 143-79
}

\section{SINOPSIS}

En el Centro Médico de Detroit se han realizado dos nuevos servicios sanitarios: un hospital de $43.000 \mathrm{~m}^{2}$ de superficie construida y capacidad para 340 camas, que cubre las necesidades de traumatología de urgencia para pacientes internos; y un instituto de sanidad, de $37.000 \mathrm{~m}^{2}$ construidos capaz para atender 550.000 visitas médicas anuales de pacientes externos y que, además, cumple una misión de medicina preventiva.

A pesar de sus distintas funciones, ambos edificios responden a un mismo esquema formal, basado en la unión de tres módulos en forma de cruz griega. En los tres primeros niveles los dos edificios se conectan mediante corredores, lo que permite mancomunar una larga serie de servicios y es, a partir de la cuarta planta, donde se independizan para satisfacer las respectivas necesidades específicas.

La configuración empleada permite, por un lado, una gran superficie de fachadas con

la correspondiente ganancia en iluminación natural $y$, por otro, reduce el volumen aparente, haciéndola más acorde con la arquitectura circundante.
El Hospital de Detroit y el Instituto de Sanidad de la Universidad Estatal de Wayne ha construido, conjuntamente, un servicio sanitario y de educación preventiva ciudadana dentro del complejo del Centro Médico de Detroit. Este servicio combina un centro traumatológico de emergencia para pacientes internos - el hospital- con capacidad para 340 camas, con un centro para pacientes externos y de enseñanza sanitaria -el instituto- que se puede realizar 550.000 visitas médicas anuales.
Ambos edificios se ubicaron en el mismo entorno, y se enlazaron a distintos niveles para conseguir importantes ahorros en la construcción. Abundando en esta idea, los dos centros comparten una larga serie de senvicios, entre los que destacan: la biblioteca, el centro educacional de conferencias, los laboratorios clínicos, la farmacia, los almacenes y los servicios de mantenimiento de las plantas. También se comparten los servicios de seguridad, transportes, archivos y comunicaciones.

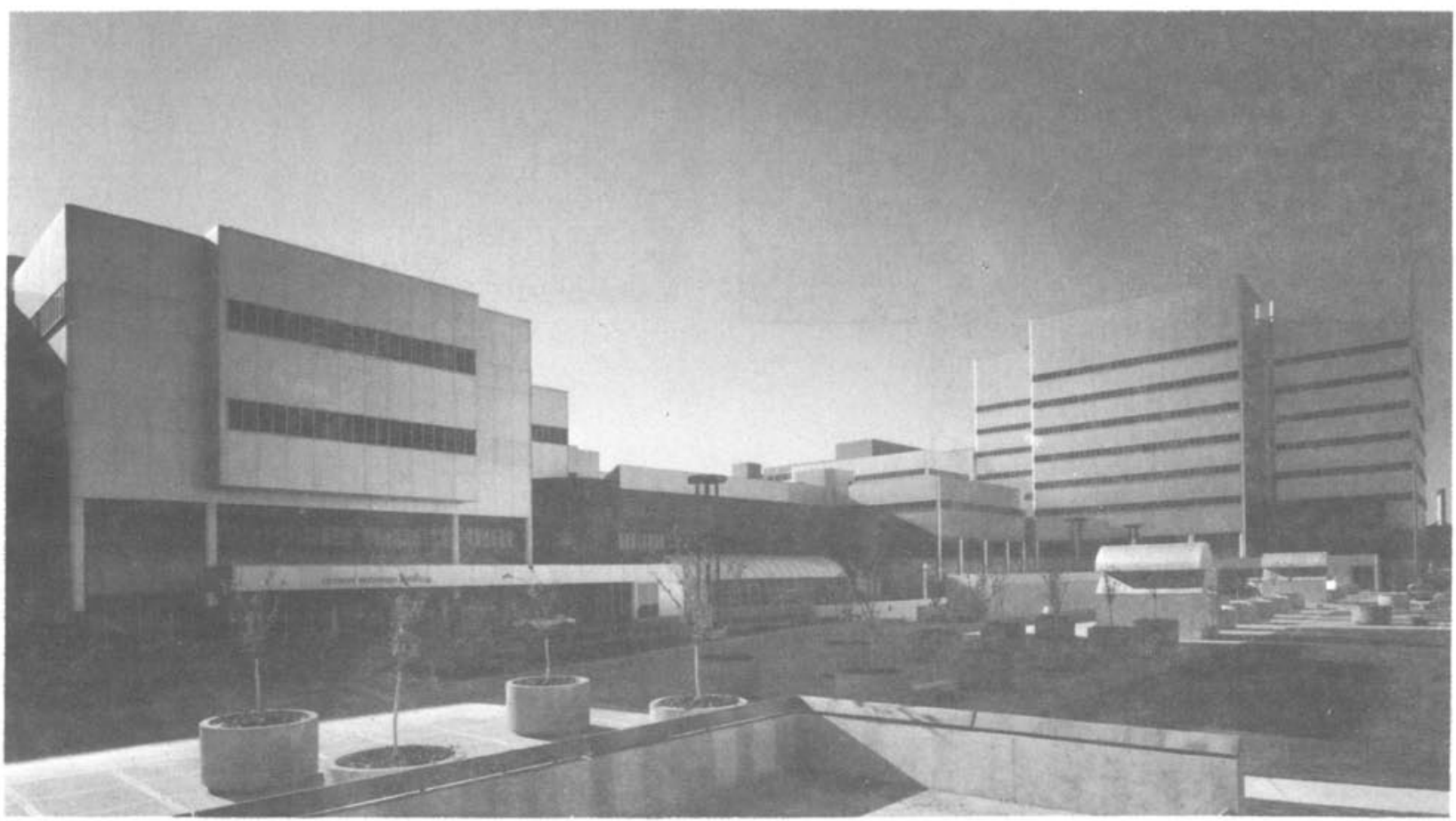



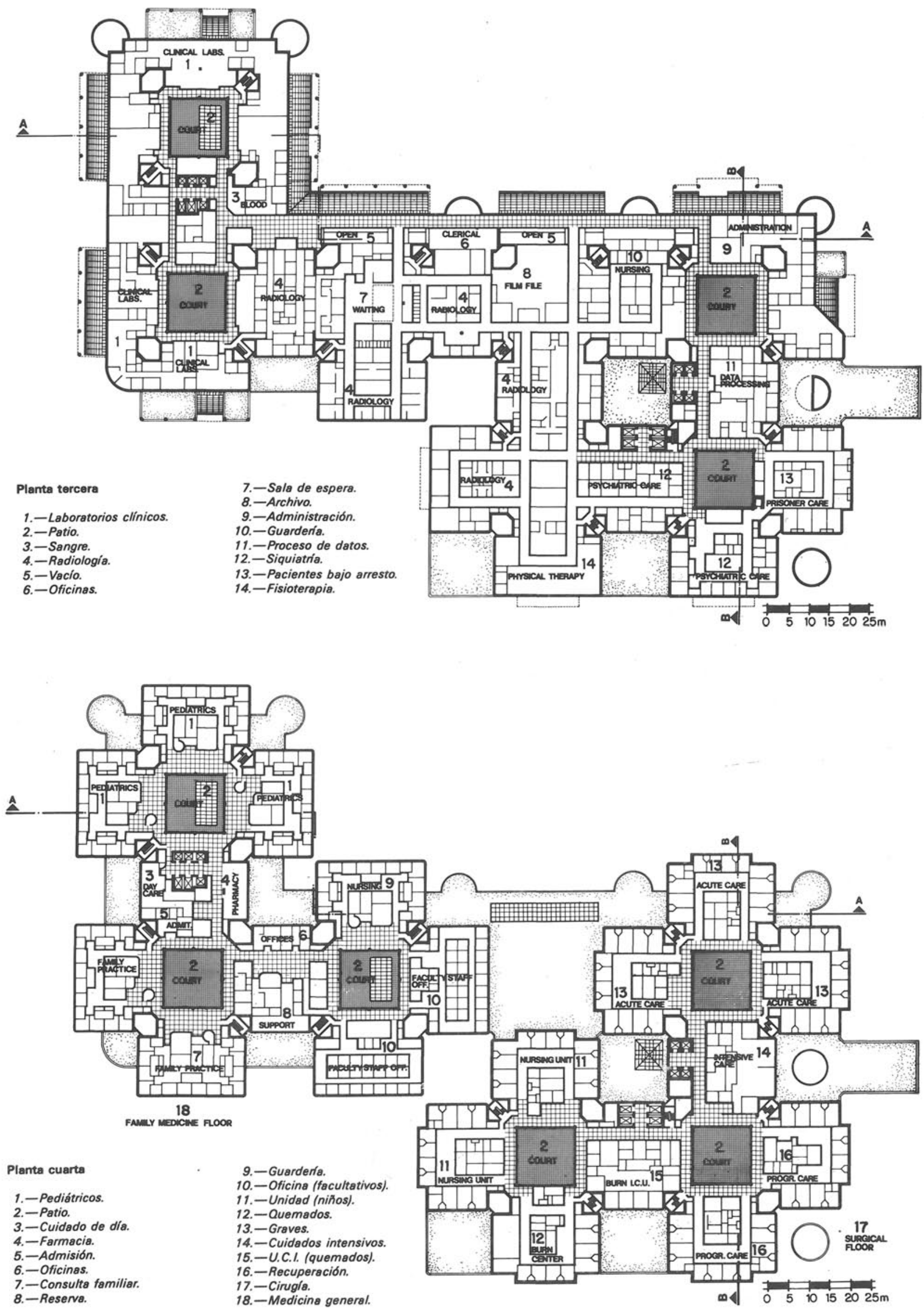
radiológico, laboratorios clínicos, farmacia y educación preventiva. La distribución del espacio en cada una de las plantas se hace mediante dos tipos de zonificación, acordes con sus distintas funciones. Las primeras, a modo de ambulatorios especializados, y situados en el interior de los módulos, agrupan cinco o seis locales que incluyen sala de tratamiento, de reuniones, zona de trabajo para el personal, etc. Las segundas, en mayor número, y situadas en el perímetro de cada módulo, son las salas individuales de consulta y reconocimiento. Estas últimas son locales prácticamente cuadrados, de $3,70 \mathrm{~m}$ de lado, que disponen una compartimentación para diferenciar sus dos funciones. Al estar situadas hacia el exterior cuentan con iluminación natural, que se complementa con otra artificial de tipo indirecto para proporcionar un ambiente agradable al paciente.

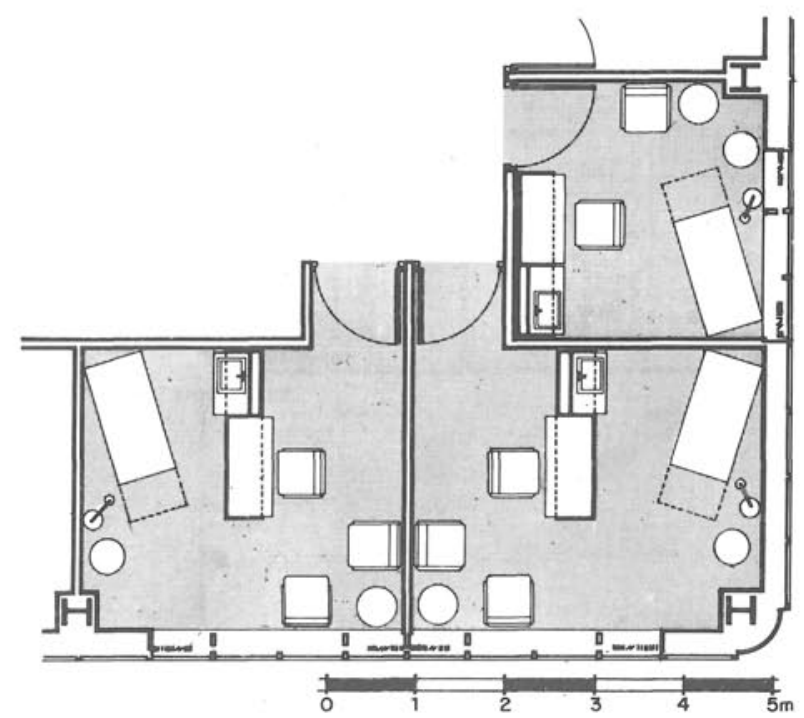

Sala de consultas y reconocimiento.

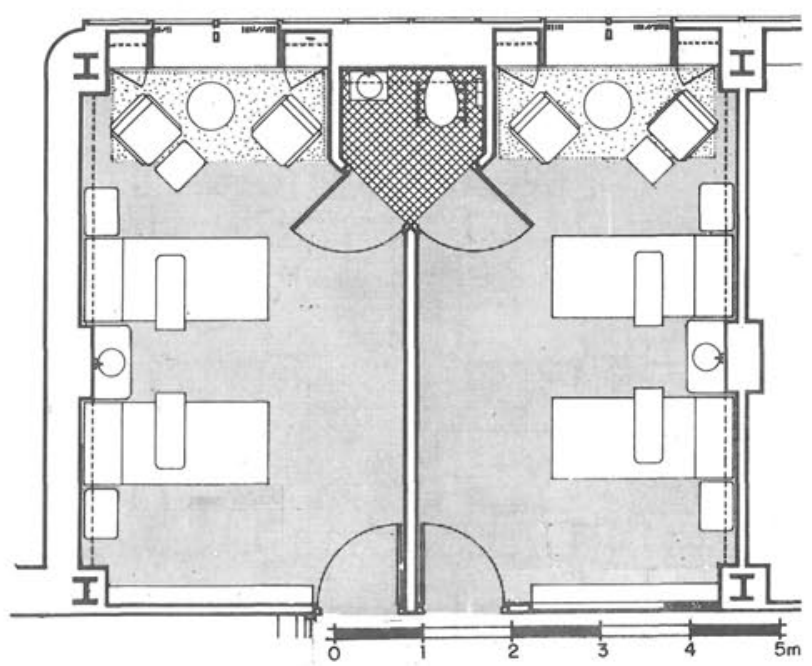

Habitación de dos camas para los enfermos.

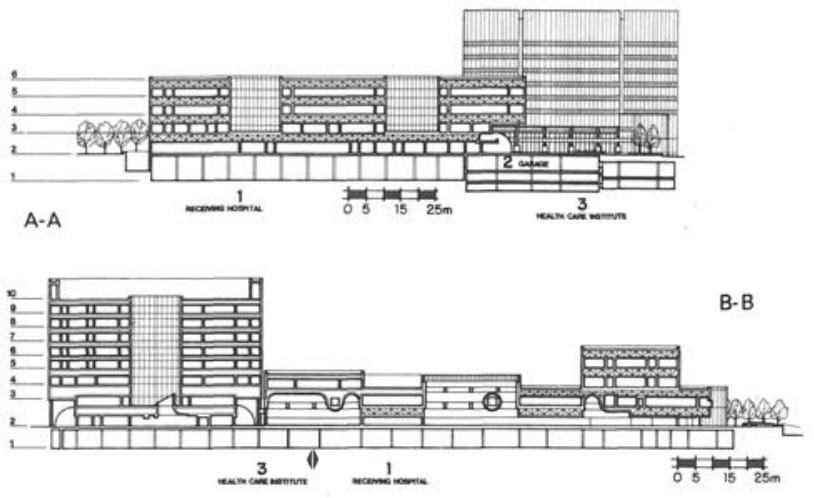

Sección A-A y sección B-B

1.-Hospital. 2.-Garaje. 3.-Instituto de la Salud.

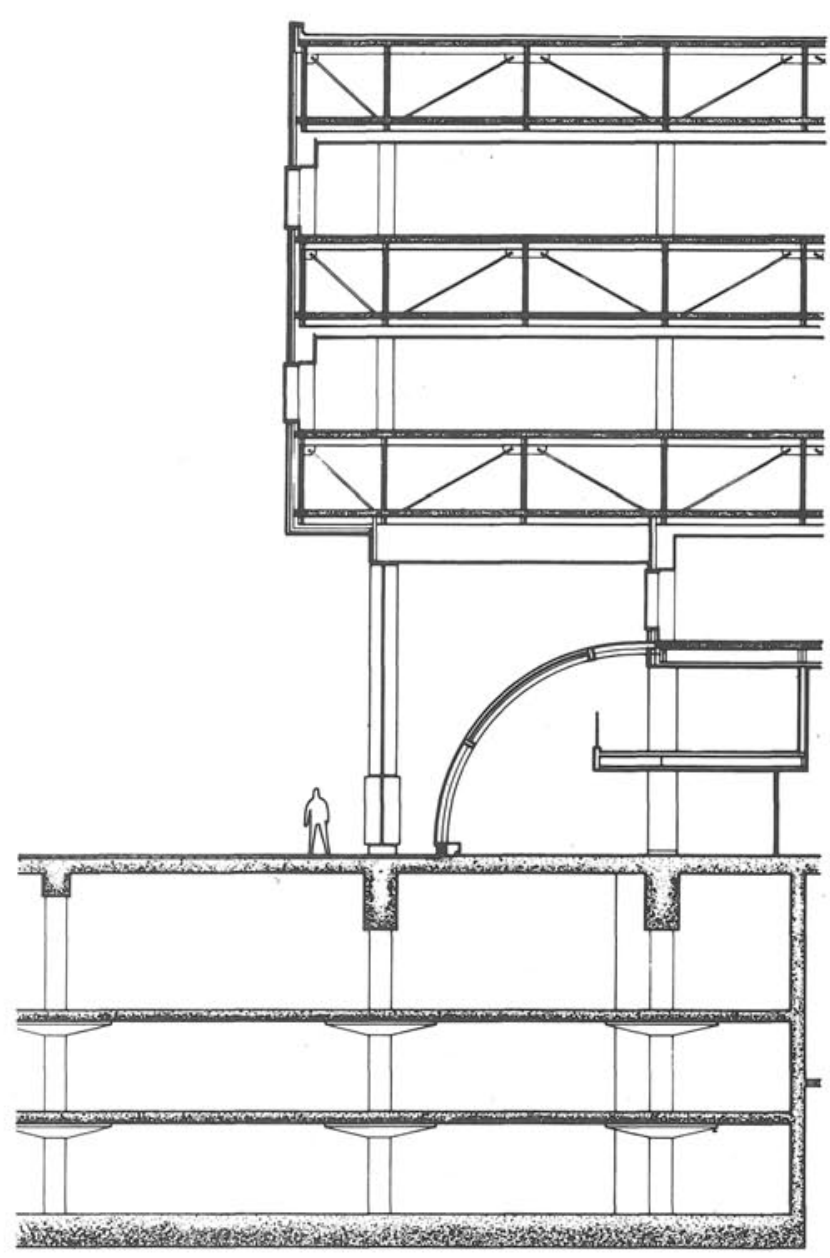

Sección (niveles).

El hospital tiene una superficie construida de $43.000 \mathrm{~m}^{2}$, además de un aparcamiento cubierto con capacidad para 630 vehículos. Sus 340 camas se han distribuido en dos plantas, y siempre en la zona perimetral de los respectivos módulos. En los brazos de cada cruz se agrupan ocho habitaciones dobles y cuatro sencillas en torno a una unidad de enfermería, con lo que la relación entre pacientes y cuidadores es prácticamente directa. Los brazos que unen las cruces se destinan, en cada planta a las unidades de cuidados 
Informes de la Construcción/337

intensivos y a los servicios especiales. Cada dos habitaciones se comparte un servicio que, junto con sus instalaciones de fontanería, está emplazado en la zona exterior. Esta disposición ha permitido utilizar mamparas en la compartimentación de espacios, en lugar de los tradicionales muros fijos, con lo que, en el futuro, se podrán hacer cambios en la distribución sin grandes obras. Las ventanas de las habitaciones, flanqueadas por los roperos de los pacientes, se situaron a corta distancia dei suelo, para permitir las vistas del exterior.

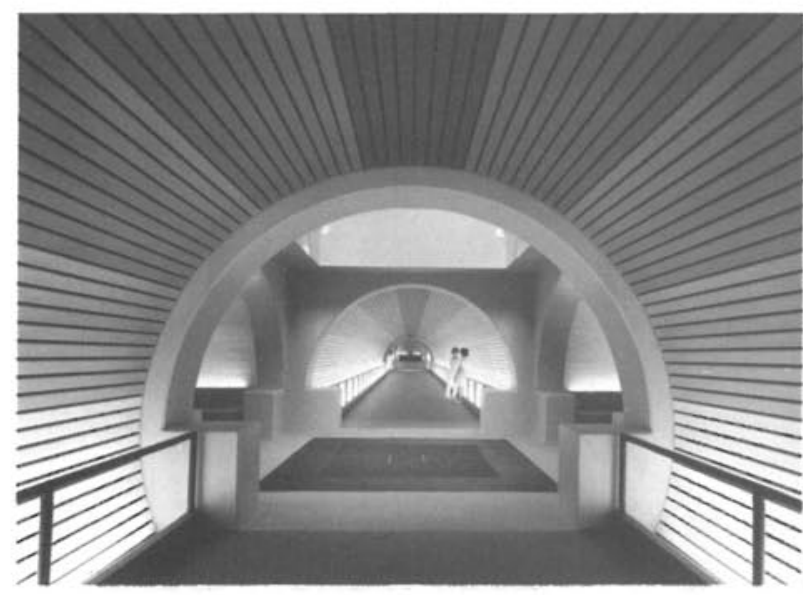

El sistema de módulos entrelazados, utilizado en la realización de los edificios, ayuda a reducir la escala de esta enorme composición proporcionando un relieve agradable al conjunto y una mayor integración en el paisaje urbano circundante. La fachada de los edificios, de paneles de aluminio de $1,50 \mathrm{~m}$ de anchura contribuye, igualmente, a disminuir el aspecto voluminoso de la construcción. El tipo de ventanas proporciona iluminación natural, pudiéndose modificar su disposición donde las necesidades del espacio interior lo requieran.

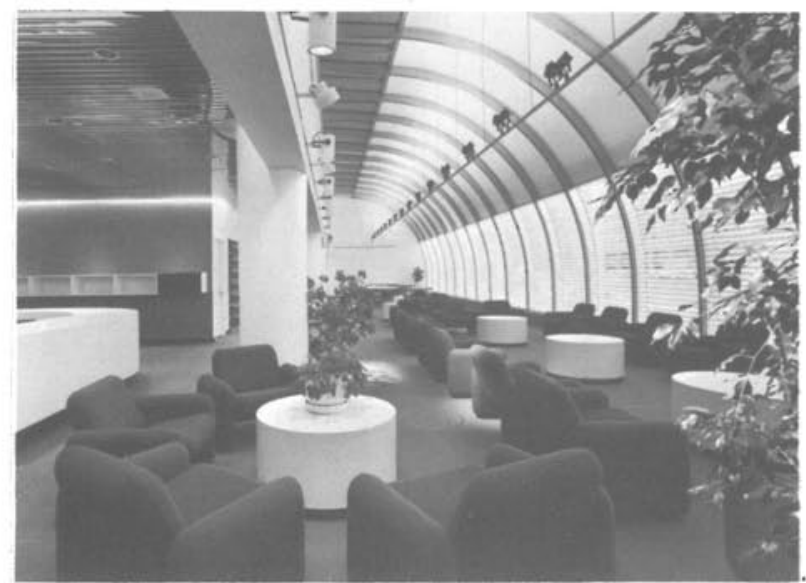

Planta, sección B-B y sección A-A
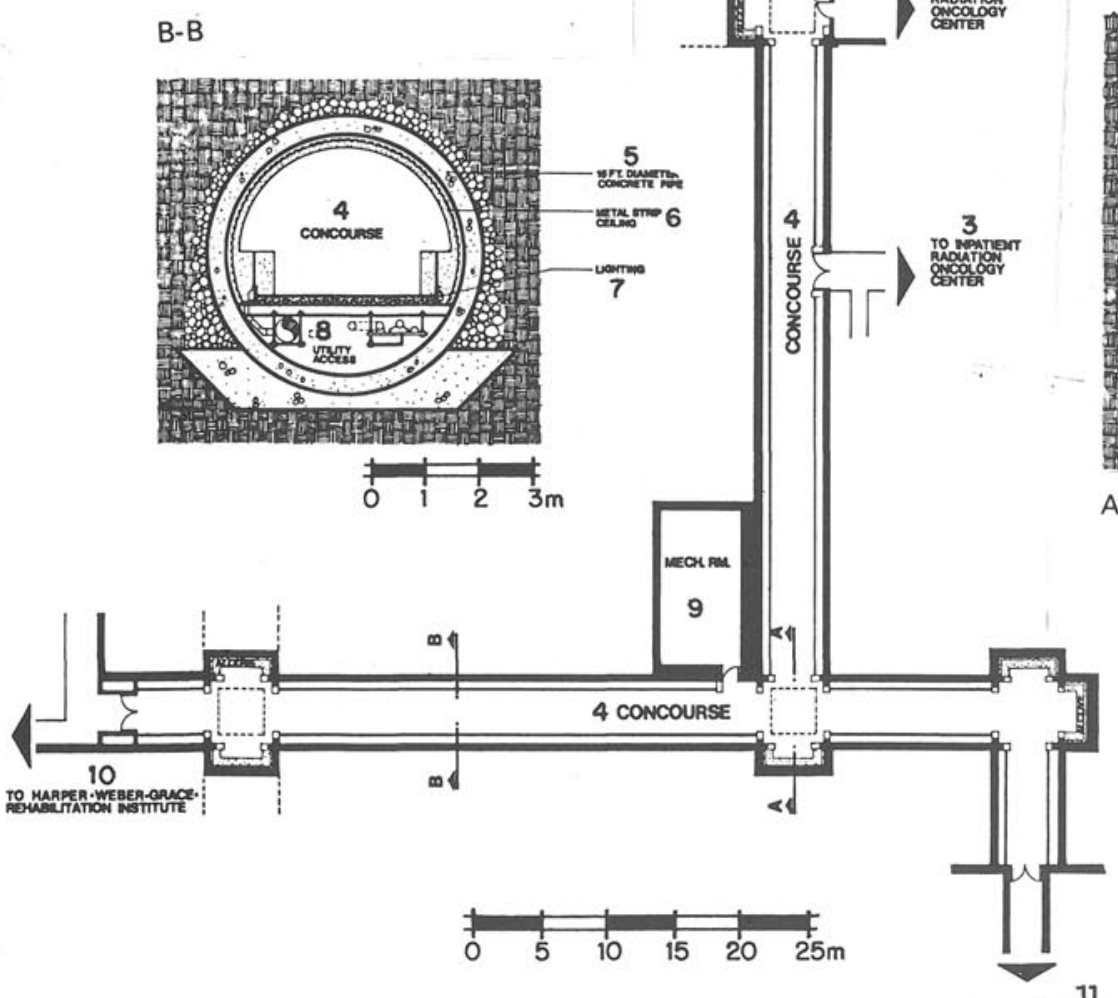

11

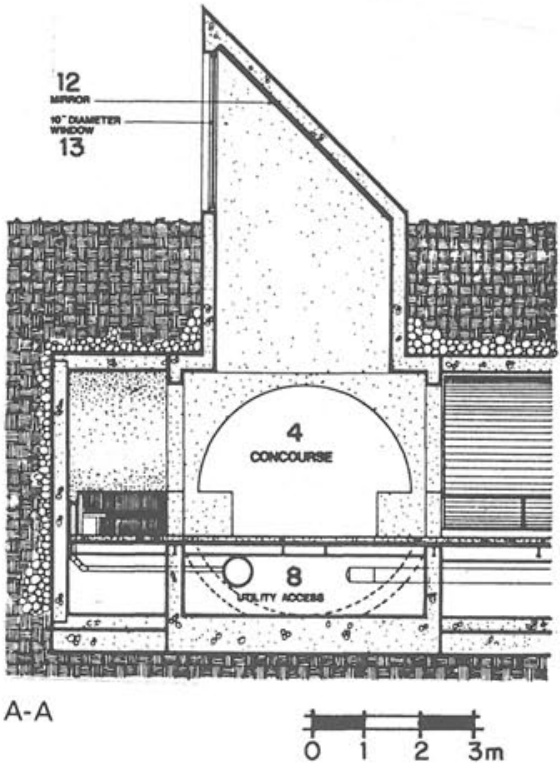

1. Al Instituto de la Universidad de Waine. 2.-Al Hospital de Detroit

3. - Al Centro de Radiación Oncológica. 4.-Pasillo.

5.-Hormigón.

6. - Techo de barras metálicas.

7.-Alumbrado.

8.-Instalaciones.

9. - Cuarto de máquinas.

9.- Cuarto de máquinas.
10.-Al Instituto de Rehabilitación

Harper-Weber-Grace.

11. Al Hospital Infantil.

12.-Espejo.

13. - Ventans 
Los dos edificios están enlazados en los tres primeros niveles, en los que se llevan a cabo la mayor parte de los servicios compartidos. A partir del cuarto nivel se separan en funciones específicas para cada uno, alcanzando cinco plantas el Hospital y nueve el Instituto.

Por varias razones se cuidó especialmente el diseño del nivel de la plaza. Contiene los servicios que
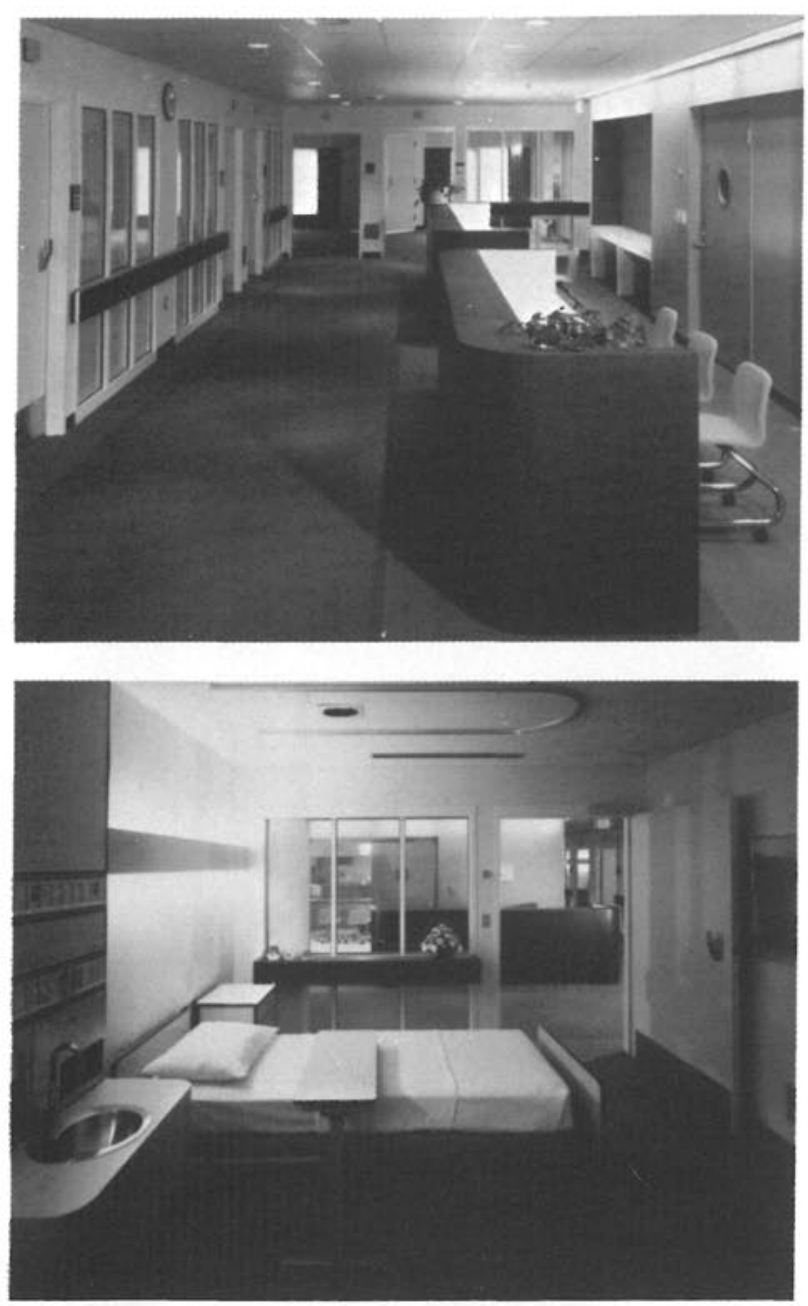

comparten el público y el personal, y proporciona la primera impresión a los que acceden al centro. Sus bóvedas de colores brillantes oponen un contrapunto a la composición general, dando un mayor equilibrio a la escala humana. La profusión de colores brillantes y materiales modernos, empleados en su decoración, le convierten en un lugar estimulante para visitantes $y$ trabajadores.
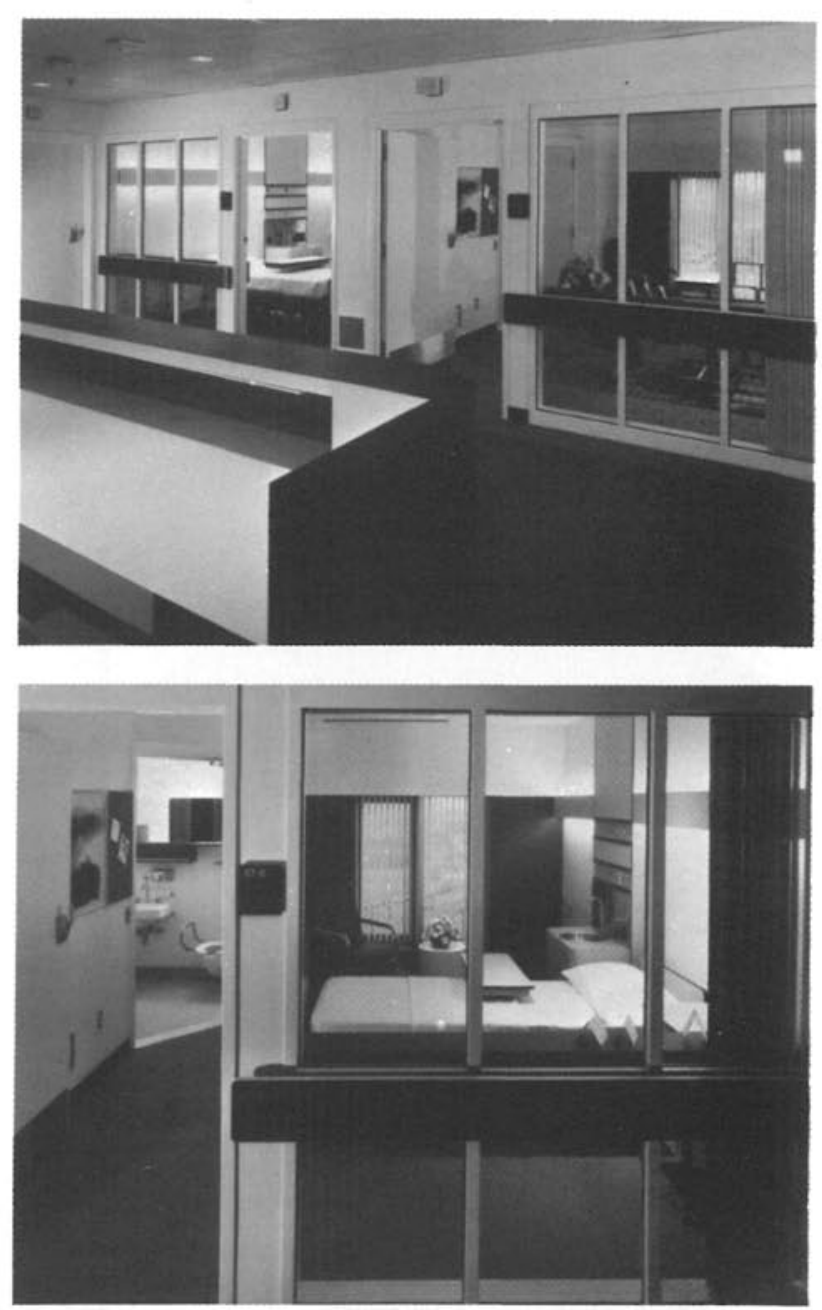

Fotos: Balthazr Korab 